\title{
Marking rejected distractors: A gaze-contingent technique for measuring memory during search
}

\author{
CHRISTOPHER A. DICKINSON and GREGORY J. ZELINSKY \\ State University of New York, Stony Brook, New York
}

\begin{abstract}
There is a debate among search theorists as to whether search exploits a memory for rejected distractors. We addressed this question by monitoring eye movements and explicitly marking objects visited by gaze during search. If search is memoryless, markers might be used to reduce distractor reinspections and improve search efficiency, relative to a no-marking baseline. However, if search already uses distractor memory, there should be no differences between marking and no-marking conditions. In four experiments, with stimuli ranging from Os and Qs to realistic scenes, two consistent data patterns emerged: (1) Marking rejected distractors produced no systematic benefit for search efficiency, as measured by reinspections, reaction times, or errors, and (2) distractor reinspection rates were, overall, extremely low. These results suggest that search uses a memory for rejected distractors, at least in those many real-world search tasks in which gaze is free to move.
\end{abstract}

Recent work has documented several relationships between visual search, the task of detecting a target among distractors, and various forms of memory. There is now good evidence suggesting that memory for previous target locations exists and can be used across search episodes (Chun \& Nakayama, 2000; Shore \& Klein, 2000). However, a question currently under debate concerns whether memory for previously inspected and rejected distractor locations can also be used to prevent the occurrence of distractor reinspections within a given search episode. Such a distractor memory has long been assumed by popular theories of search (Treisman \& Gelade, 1980; Wolfe, 1994). The clearest expression of this belief is the prediction of a 2:1 ratio of linear target-absent to target-present search slopes by serial exhaustive search models - a prediction requiring a record of rejected distractor locations. This widely held assumption, however, has been challenged by Horowitz and Wolfe (1998), who showed that the random relocation of objects in a search task did not impair search efficiency, relative to when the objects remained stationary. Horowitz and Wolfe (1998) concluded from this finding that the search process is amnesic and does not use memory for rejected distractors (but see Dickinson \& Zelinsky, 2002, and Mühlenen, Müller, \& Müller, 2003, for alternative explanations).

Although subsequent investigations (Horowitz \& Wolfe, 2001, 2003; Woodman, Vogel, \& Luck, 2001) have supported the conclusion that search does not use memory,

This work was supported by grants from the NSF (ITR-0082602), NIMH (R01-MH63748), and ARO (DAAD19-03-1-0039) to G.J.Z. Correspondence concerning this article should be addressed to G. J. Zelinsky, Department of Psychology, State University of New York, Psych B Bldg., Room 240, Stony Brook, NY 11794-2500 (e-mail: gregory .zelinsky@stonybrook.edu). other evidence has accumulated that supports the opposite conclusion (Kristjánsson, 2000; Müller \& Mühlenen, 2000). Many of the researchers arguing for the existence of distractor memory have used eye movement paradigms to make their case (Aks, Zelinsky, \& Sprott, 2002; McCarley, Wang, Kramer, Irwin, \& Peterson, 2003). For example, Klein and MacInnes (1999) found a bias against directing saccades to distractor locations visited by gaze within the last two fixations, a pattern that they interpreted as evidence for distractor memory. An eye movement paradigm also allows one to quantify distractor memory use in terms of a proportion of rejected distractors that are refixated during search. Studies in which this method has been used have shown that the observed refixation rate is lower than the rate expected for a memoryless model, again suggesting that distractor memory is used during a search task (Gilchrist \& Harvey, 2000; Peterson, Kramer, Wang, Irwin, \& McCarley, 2001).

\section{Using the World as an External Memory}

We introduce a methodology that builds on earlier refixation-based assessments of memory during search. Our technique provides observers with a visible record of fixated objects by making gaze-contingent changes to the search display. For example, if a salient red box is drawn around a search object each time gaze shifts away (Figure 1), the accumulation of these markers during a search trial will create a sort of enduring external memory (O'Regan, 1992) of the observer's search history. We compared several of these marking conditions with no-marking baselines to determine whether differences exist in search efficiency or distractor refixation rate. If there exists no internal memory for rejected search distractors, we should find more distractor refixations in the no-marking condition, as compared with conditions in which markers are externally available to assist search. However, if observ- 


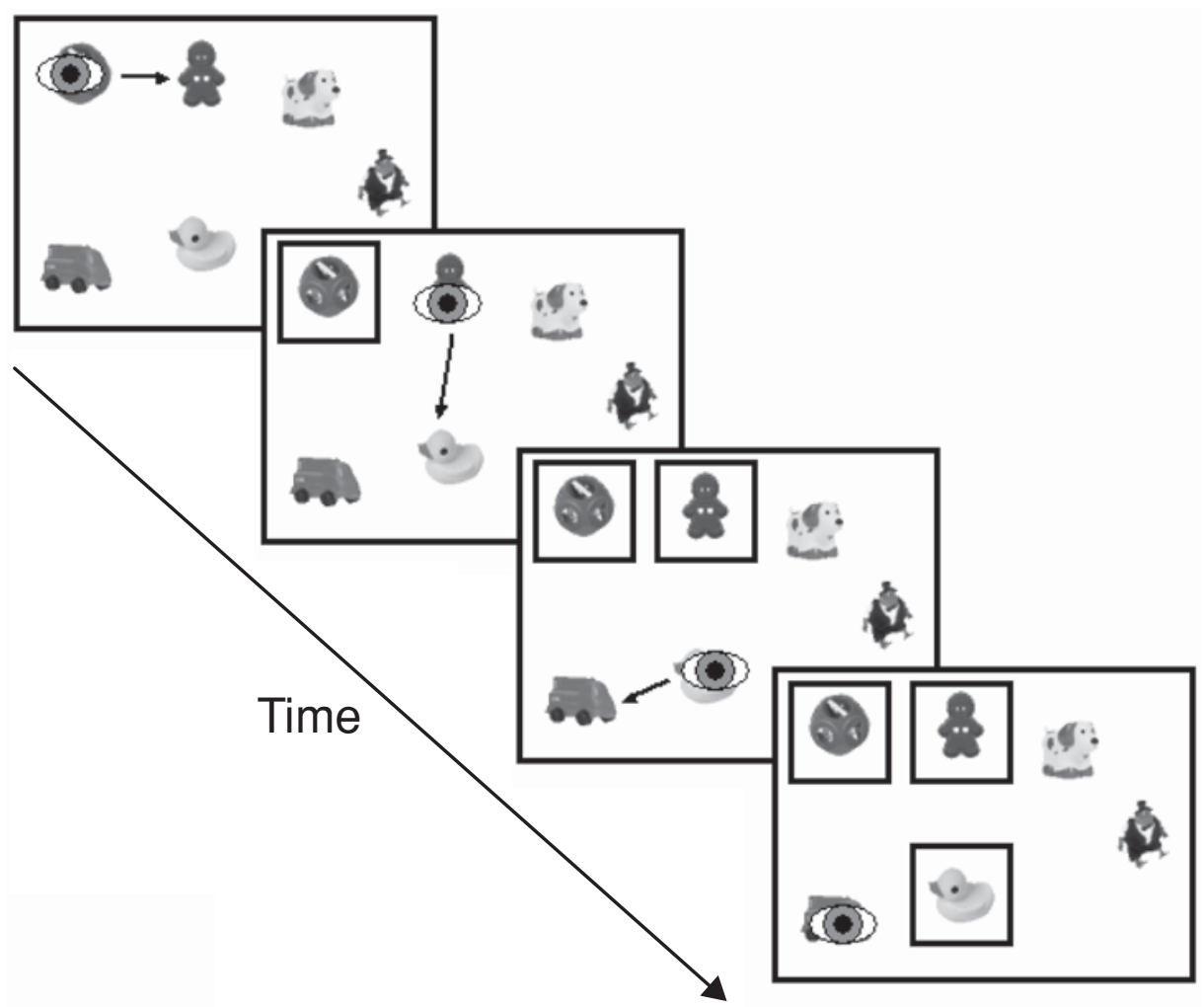

Figure 1. A schematized illustration of gaze-contingent explicit marking. The cartoon eye in each panel represents an observer's gaze position. Note that marking occurs as gaze shifts to a new display object.

ers have a good internal memory for distractors and use it to avoid reinspecting objects, external markers will be redundant and should, therefore, not improve search efficiency or lower distractor refixation rates. We also compared refixation rates with a memoryless random fixation model. Here, we predicted that if observers were using either an internal or an external distractor memory during search, the observed refixation rates should be lower than those generated by the memoryless model.

\section{METHOD}

Because one's reliance on distractor memory might reasonably vary with the difficulty of the search task, we used four very different stimulus sets in this study. The target in Experiment 1 was an $\mathrm{O}$ embedded in rotated Q-like distractors. In Experiment 2, observers were asked to search for a real-world object in an array of object distractors. Experiment 3 was a Where's Waldo search task, and Experiment 4 had observers search for a tank target in fully realistic landscape scenes. The methodological details common to all four experiments are described below; details specific to each experiment are described in the Appendix.

\section{Participants}

In both Experiments 1 and 2, 12 observers were tested; in both Experiments 3 and 4, 10 observers were used. No observer participated in more than one experiment, and all had normal or correctedto-normal vision.

\section{Apparatus}

Eye movement and manual data were collected using the EyeLink II eye-tracking system (SR Research). Eye position was sampled at $500 \mathrm{~Hz}$, the system's spatial resolution was estimated to be $0.2^{\circ}$, and changes in gaze position were available to the computer controlling the display within $8 \mathrm{msec}$. Search displays subtended $27^{\circ} \times 20^{\circ}$ $(800 \times 600$ pixels $)$ and were presented on a 19 -in. CRT monitor at a refresh rate of $100 \mathrm{~Hz}$. Head position and viewing distance were fixed with a chinrest, and all responses were made with a GamePad controller attached to the computer's USB port. Judgments were made with the left and right index-finger triggers; trials were initiated with a button operated by the left thumb.

\section{Procedure}

The displays in Experiments 1 and 2 consisted of discrete objects appearing on a black background; the displays in Experiments 3 and 4 consisted of scenes that could not be easily decomposed into discrete objects. These two different display types required different methods of marking fixated locations. In the discrete-object displays, each object was centered in an invisible bounding circle that was used to assign a fixation to an object. Fixated objects were tagged by replacing them with a marked version of the object, and fixated objects were marked only if gaze duration within the bounding circle exceeded a minimum fixation threshold (see the Appendix for details). Objects were marked after gaze moved outside of the bounding circle, with each marker remaining visible for the duration of the trial. In the scene-based search tasks, fixated locations were marked if the distance between the current fixation and the last marked fixation exceeded a minimum distance threshold and a minimum fixation duration criterion was satisfied. The markers 
used in these experiments were semitransparent color overlays $\left(1^{\circ}\right.$ squares) centered on the location of the fixation. Figure 1 illustrates the marking process; approximations of marked and unmarked displays are shown in Figure 2 for each experiment.

Search displays remained visible until a target-present or targetabsent response. Marking and no-marking trials were blocked and counterbalanced across participants; target condition (present or absent) was interleaved. Practice trials preceded each block of trials. Participants were asked to respond as quickly as possible without sacrificing accuracy, and were told that the markers would provide a valid history of their fixated objects and might therefore prove useful to their search.

\section{RESULTS}

\section{Reinspection Analysis}

Before we could derive the distractor reinspection rate, we first had to convert fixations into inspections in order to deal with cases in which objects were multiply fixated within a single viewing. For Experiments 1 and 2, this involved collapsing sequential fixations falling within the same bounding circle into a single inspection. In the rare event that a fixation did not fall within a bounding circle, it was assigned to the nearest object and treated as any other fixation for the purpose of calculating an inspection.
Because the displays in Experiments 3 and 4 were scenes, rather than discrete-object arrays, we subdivided each display into a 48 -cell grid of $100 \times 100$ pixel squares $\left(3.4^{\circ} \times\right.$ $3.3^{\circ}$ ) and then collapsed sequential fixations falling within the same cell into a single inspection. Reinspections were defined as any inspections falling within a previously inspected grid location. We excluded immediate refixations from these analyses, since the cause of these refixations has been attributed to the incomplete processing of objects (Hooge \& Erkelens, 1998) and, therefore, cannot be unambiguously attributed to memory failure.

Basic search data, including the number of inspections for all four experiments, are shown in Table 1. Turning first to the gaze inspection data, two general patterns are clear. First, the number of inspections varied considerably across our search tasks, ranging from 36 in Experiment 1 to 2.5 in Experiment 2. Second, in only two cases (out of 12 comparisons) was a significant difference observed between the marking and the no-marking conditions (all other $p \mathrm{~s}>.05)$.

Both of these patterns can also be seen in the reinspection data (Figure 3; solid bars). Reinspection rates in the target-present trials (top) were uniformly low. More-
A

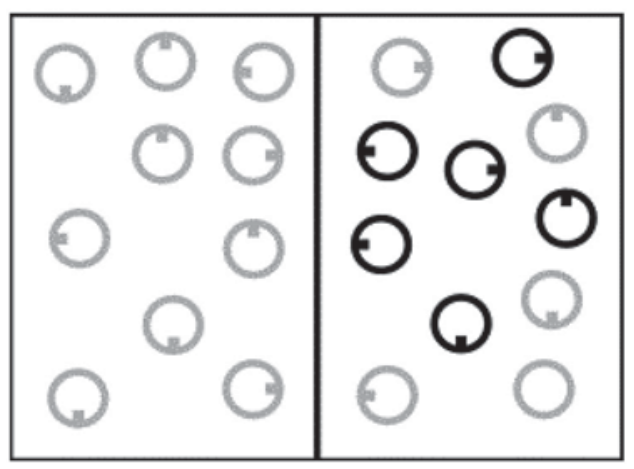

C

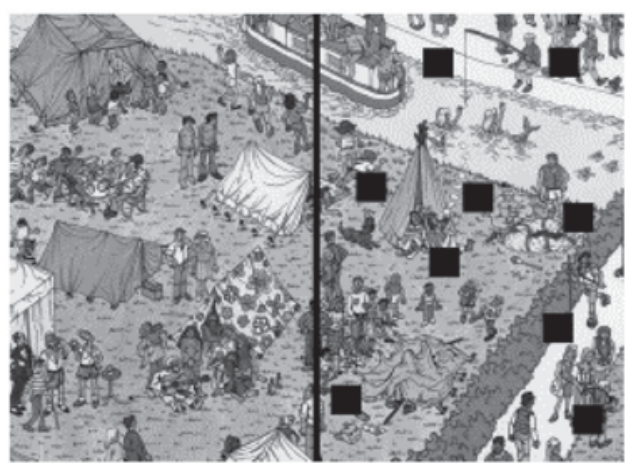

B

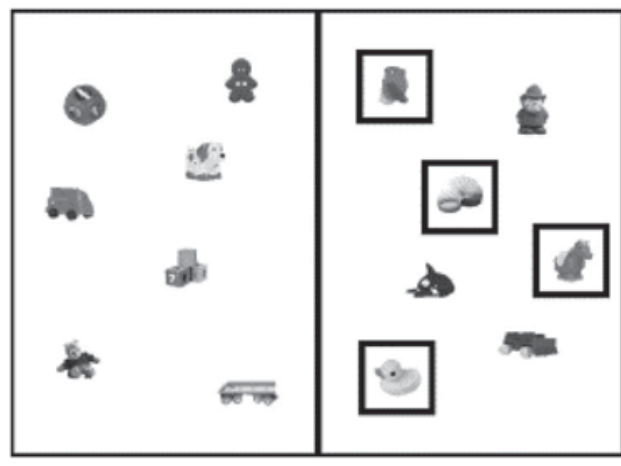

D

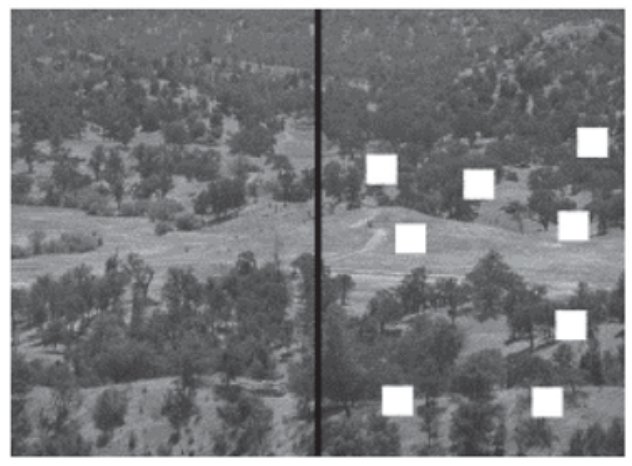

Figure 2. Examples of search stimuli from Experiments 1-4. The left side of each panel illustrates display conditions in a no-marking trial or prior to the addition of any markers in a marking trial (i.e., at the start of the trial); the right half illustrates display conditions after several fixations during marking trials. Shown are representations of the $O$ search task (A, schematic), the object search task (B), the Where's Waldo task (C, schematized markers), and the tank search task (D, schematized markers). Note that the actual stimuli were in color. See the Appendix for details regarding the specific marking criteria and display conditions used in each experiment. 
Table 1

\begin{tabular}{|c|c|c|c|c|}
\hline \multirow[b]{2}{*}{ Experiment } & \multicolumn{2}{|c|}{ Present } & \multicolumn{2}{|c|}{ Absent } \\
\hline & Marking & No Marking & Marking & No Marking \\
\hline \multicolumn{5}{|c|}{ Number of Inspections } \\
\hline $1(7)^{\dagger}$ & 5.2 & 5.0 & 9.2 & 9.6 \\
\hline $1(21)$ & 12.8 & 12.1 & 22.5 & $24.5^{*}$ \\
\hline $1(35)$ & 18.0 & 18.1 & 34.1 & 36.0 \\
\hline 2 & 2.5 & $3.1^{*}$ & 6.0 & 5.8 \\
\hline 3 & 8.4 & 8.8 & 27.3 & 26.1 \\
\hline $4 \ddagger$ & 4.7 & 4.4 & 19.1 & 17.3 \\
\hline \multicolumn{5}{|c|}{ Reaction Time } \\
\hline $1(7)^{\dagger}$ & 2,296 & 2,123 & 3,183 & 3,297 \\
\hline $1(21)$ & 4,178 & 3,909 & 7,458 & 7,869 \\
\hline $1(35)$ & 5,659 & 5,395 & 11,237 & 11,480 \\
\hline 2 & 1,118 & 1,217 & 1,663 & 1,606 \\
\hline 3 & 3,139 & 3,197 & 9,264 & 8,364 \\
\hline $4 \ddagger$ & 2,036 & 1,823 & 6,891 & 5,941 \\
\hline \multicolumn{5}{|c|}{ Percentage of Errors } \\
\hline $1(7)^{\dagger}$ & 2.5 & 2.5 & 0.6 & 0.8 \\
\hline $1(21)$ & 18.6 & 13.9 & 0.3 & 0.6 \\
\hline $1(35)$ & 18.9 & 18.6 & 0.6 & 0.3 \\
\hline 2 & 7.2 & 6.7 & 0.8 & 1.9 \\
\hline 3 & 5.8 & 11.2 & 2.1 & 1.4 \\
\hline $4+$ & 12.9 & $5.4^{*}$ & 1.3 & 2.5 \\
\hline
\end{tabular}

Note-Initial fixations are not included in the inspection data. * *Significant differences ( $p<.05$, two-tailed $t$ test) between the marking and the nomarking conditions. †The numbers 7,21 , and 35 refer to the set sizes in Experiment 1. \$Target-present data for Experiment 4 represent the mean of both the large and the small target types.

over, except for a small (2\%) marker benefit in the Experiment 135 set size data $[t(11)=-5.60, p<.001]$ and a small $(2 \%)$ marker cost in the Experiment 4 data $[t(11)=3.98, p=.003]$, reinspection rates did not differ between the marking and the no-marking conditions $(p s>.05)$. Similar patterns were observed in the targetabsent reinspection data (bottom). Although reinspections were more common in the target-absent data, relative to the target-present data, as would be expected given the greater number of inspections made during target-absent trials (Table 1), no marker benefits were found in Experiments $2-4(p s>.05)$.

The target-absent data from Experiment 1 were more mixed. Here, we found a small (approximately 5\%) but significant main effect of marking $[F(1,11)=26.41, p<$ $.05]$. However, given the size of this effect and its failure to interact with set size $[F(2,22)=0.84, p>.05]$, we attribute these differences to the external markers' preventing the one or two terminal reinspections that often precede an exhaustive target-absent search response and do not take them as evidence for memory failure. Note also that reinspections decreased slightly with increasing set size $[F(2,22)=5.80, p<.05]$. Although this trend may suggest that search is more amnesic at smaller set sizes, we think that it is more likely that the larger amplitude saccades required by sparser displays resulted in greater saccade error and the occasional inadvertent reinspection of distractors. Taken together, the overall patterns of results from all four experiments suggest that the observers had an internal representation of their search history and that this memory was nearly as effective as external markers in preventing distractor reinspections.

The reinspection rates shown in Figure 3 are clearly low, but are they lower than what would be expected from chance? We addressed this question by comparing the behavioral reinspection rates with the rates predicted by a memoryless search model. Simulations were conducted on an observer-by-observer and trial-by-trial basis, with the number of behavioral inspections on a given trial $(n)$ determining the number of search objects selected by the model. For each trial, the model therefore randomly selected, with replacement and without regard for display characteristics, $n$ "fixations" from the set of locations corresponding to object positions in the display. ${ }^{1}$ Reinspections occurred whenever the model selected the same object more than once during a trial. ${ }^{2}$

These simulated reinspection rates (hatched bars) are plotted alongside the behavioral rates in Figure 3. Behavioral reinspection rates in Experiments 3 and 4 and all three of the set size conditions in Experiment 1 were much lower than what would be expected from a memoryless search model in both the target-present and the targetabsent data $(p s<.05)$. Target-present reinspection rates in Experiment 2 were uninterpretable, due to the small number of fixations accompanying search in that condition; however, a small but significant difference was found in the target-absent data $[t(11) \geq 5.40, p<.001]$. These below-chance reinspection rates provide converging evidence for the use of distractor memory during search. If the observers had not used their distractor memory in the no-marking condition, or if they had ignored the markers in the marking condition, their reinspection rates should have been at chance, as defined by the memoryless model. This was clearly not the case.

\section{Manual Data}

We know from the eye data analyses that reinspection rates did not differ substantially between the marking and the no-marking search conditions, but were there differences in overall search efficiency? Table 1 shows the reaction time (RT) and error data from both display conditions, with significant differences between marking and no-marking data indicated by an asterisk. Of these 24 comparisons, only 1 produced a significant marking difference, and this difference described a marker cost, rather than a benefit. ${ }^{3}$ Moreover, search slopes in the Experiment 1 target-present (absent) data were 120 (288) msec/ object in the marking condition and 117 (292) msec/object in the no-marking condition, not significant differences $(p \mathrm{~s}>.05)$. Consistent with the reinspection analyses, having visible markers available produced no evidence for improvement in search efficiency.

One potential concern raised by the marking paradigm is that the marker onsets might be capturing the observers' attention and, therefore, interfering with their search. It might be the case that a marker benefit was being offset by a distracting effect of the marker. To look for such a trade-off, we analyzed the frequency of immediate rein- 


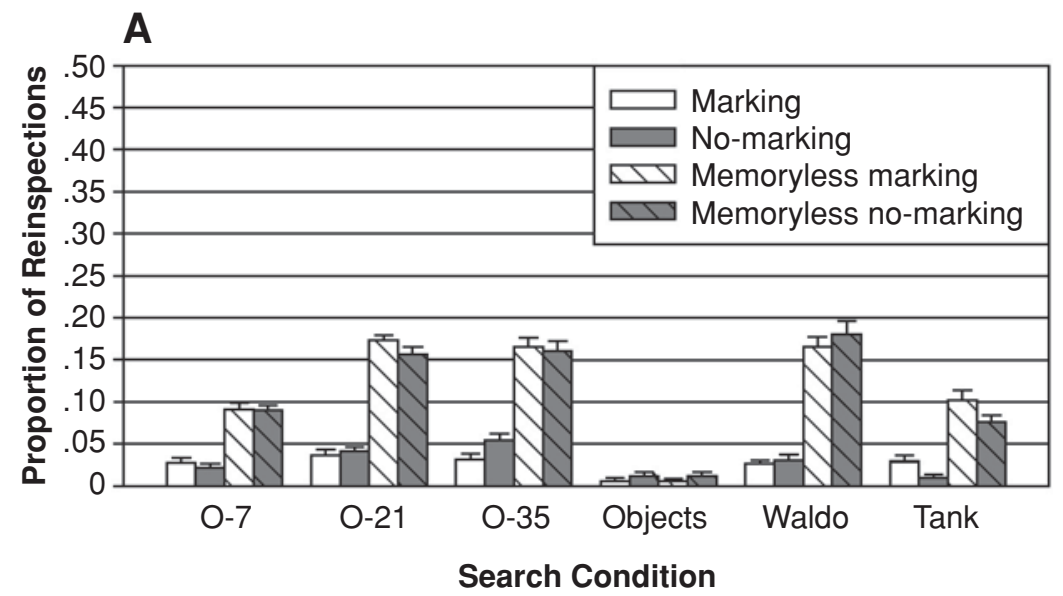

B

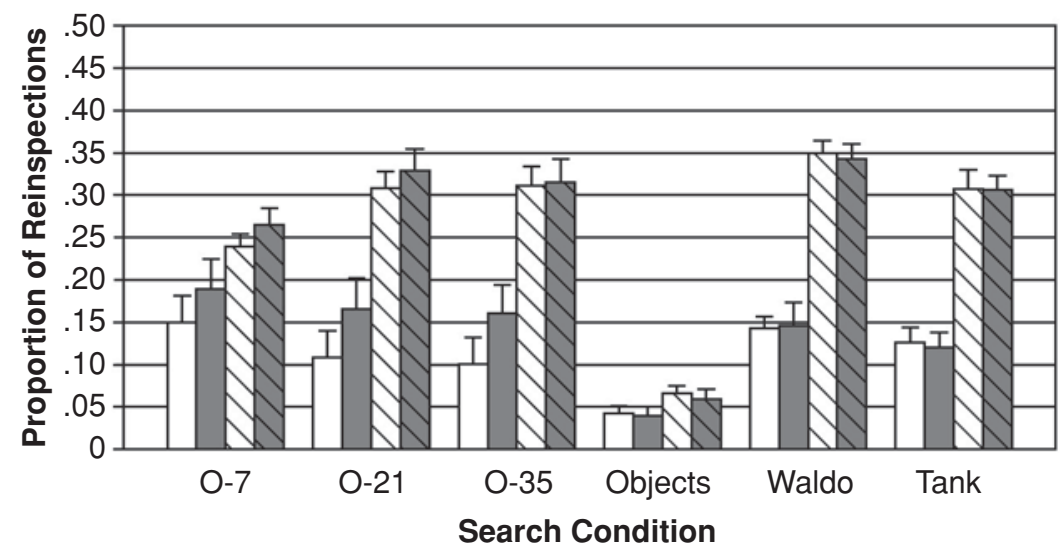

Figure 3. Proportions of distractor reinspections under marking (white bars) and no-marking (gray bars) conditions for the $\mathrm{O}(\mathrm{O}-7, \mathrm{O}-21$, and $\mathrm{O}-35$ refer to the three set sizes), object, Waldo, and tank search tasks. Hatched bars show the corresponding simulated reinspection rates from our memoryless model. Target-present data are shown in panel A, and target-absent data are shown in panel B. Note that we report simulated data for both the marking and the no-marking conditions. Although this manipulation would not be expected to affect a memoryless model, because our simulations were based on the total number of inspections in the behavioral data, slight differences in the behavioral inspection rate between the marking and the no-marking conditions (see Table 1) resulted in slightly different simulation results.

spections in our data. If marker onsets were capturing attention in our tasks, one would expect frequent and immediate gaze shifts back to the most recently marked object (Theeuwes, Kramer, Hahn, Irwin, \& Zelinsky, 1999). Comparing the frequency of immediate reinspections in the marking and the no-marking conditions revealed no evidence for this hypothesis. Only in the O-Q task was a significant difference observed $[t(11)=4.87, p<.05]$, but this difference was in the opposite direction of what would be predicted from an attention capture effect (i.e., $4.3 \%$ immediate reinspections without a marker, $3.1 \%$ immediate reinspections with a marker). No other differences approached significance, suggesting that the scarcity of marker effects on search efficiency was probably not caused by marker benefits being negated by marker onset costs. ${ }^{4}$

\section{DISCUSSION}

Many of our day-to-day search tasks take place in complex environments, with numerous potential locations requiring inspection. Clearly, using memory of one's search history could facilitate many of these searches, particularly when the target of the search is not present. In this study, we introduced a gaze-contingent paradigm to explicitly manipulate the availability of information about previously fixated search distractors. The results of four experiments converged on a single conclusion: Providing a visible record of one's search history had little or no impact on either search efficiency or reinspection frequency. Moreover, distractor reinspections were found to be quite infrequent and clearly below chance in both object-based and scene-based search tasks. 


\section{What About Memoryless Search?}

Our findings have important implications for the question of whether distractor memory is used during search. Recent years have seen mounting evidence arguing against Horowitz and Wolfe's (1998) claim that search has no memory (Aks et al., 2002; Gilchrist \& Harvey, 2000; Kristjánsson, 2000; McCarley et al., 2003; Müller \& Mühlenen, 2000; Peterson et al., 2001). The present data add to this growing chorus of studies by showing no meaningful benefit to search from a valid and readily available external memory of rejected distractors. Rather than search being memoryless, our data suggest the opposite conclusion: that a nearly perfect memory exists for where one has searched and that this memory is used optimally to avoid distractor reinspections. However, our data do not require the representation of many discrete rejected distractors or location tags, constructs traditionally associated with distractor memory. We believe that distractor memory might, instead, take the form of a more abstracted search plan (see Peterson, Beck, \& Vomela, 2004, for a similar proposal). If observers are able to represent the path that they follow during their search, it follows that they should demonstrate evidence for distractor memory well beyond the memory capacity limits suggested by recent studies (McCarley et al., 2003; Peterson et al., 2001). Future work will explore this possibility.

Regardless of its form, it appears that our observers utilized their internal representations sufficiently well that the provision of external markers was informationally redundant and not helpful to their search. The fact that this pattern was observed across four search tasks that varied markedly in difficulty and stimulus complexity makes this finding all the more compelling. Our finding is also consistent with recent work on visual neglect in which a cancellation test was used (Wojciulik, Rorden, Clarke, Husain, \& Driver, 2004). The fact that patients showed marker benefits in their neglected hemifield suggests that the normally high-functioning internal marking system may malfunction in people with neglect. Finally, although our data do not speak to the question of whether distractor memory is used in the absence of eye movements, given that eye movements are made in the vast majority of realworld search tasks and that these eye movements reflect distractor memory, the conclusion follows that this memory is used during most of our day-to-day search tasks. This suggests that cases in which distractor memory is not used to facilitate search are more likely the exception, rather than the rule.

\section{REFERENCES}

Aks, D. J., Zelinsky, G. J., \& Sprott, J. C. (2002). Memory across eye-movements: $1 / f$ dynamic in visual search. Nonlinear Dynamics, Psychology, \& Life Sciences, 6, 1-25.

Chun, M. M., \& Nakayama, K. (2000). On the functional role of implicit visual memory for the adaptive deployment of attention across scenes. Visual Cognition, 7, 65-81.

Dickinson, C., Chen, X., \& Zelinsky, G. (2003). Explicitly marking rejected distractors in an overt visual search task [Abstract]. Journal of Vision, 3, 158a.

Dickinson, C., \& Zelinsky, G. (2002). Sitting and waiting for the tar- get: A strategy for searching dynamic displays. Abstracts of the 43rd Annual Meeting of the Psychonomic Society, 7, 94.

Gilchrist, I. D., \& HARvey, M. (2000). Refixation frequency and memory mechanisms in visual search. Current Biology, 10, 1209-1212.

Hooge, I. T. C., \& ERKelens, C. J. (1998). Adjustment of fixation duration in visual search. Vision Research, 38, 1295-1302.

Horowitz, T. S., \& Wolfe, J. M. (1998). Visual search has no memory. Nature, 394, 575-577.

Horowitz, T. S., \& Wolfe, J. M. (2001). Search for multiple targets: Remember the targets, forget the search. Perception \& Psychophysics, 63, 272-285.

Horowitz, T. S., \& Wolfe, J. M. (2003). Memory for rejected distractors in visual search? Visual Cognition, 10, 257-287.

KLEIN, R. M., \& MACINNES, W. J. (1999). Inhibition of return is a foraging facilitator in visual research. Psychological Science, 10, 346-352.

KRISTJÁnsson, A. (2000). In search of remembrance: Evidence for memory in visual search. Psychological Science, 11, 328-332.

McCarley, J. S., Wang, R. F., Kramer, A. F., Irwin, D. E., \& PeterSON, M. S. (2003). How much memory does oculomotor search have? Psychological Science, 14, 422-426.

Mühlenen, A. von, Müller, H. J., \& Müller, D. (2003). Sit-andwait strategies in dynamic visual search. Psychological Science, 14, 309-314.

Müller, H. J., \& MüHlenen, A. vON (2000). Probing distractor inhibition in visual search: Inhibition of return. Journal of Experimental Psychology: Human Perception \& Performance, 26, 1591-1605.

O'REgan, J. K. (1992). Solving the "real" mysteries of visual perception: The world as an outside memory. Canadian Journal of Psychology, 46, 461-488.

Peterson, M. S., Beck, M. R., \& Vomela, M. (2004, May). The guidance of attention by retrospective and prospective memory during visual search. Paper presented at the 4th Annual Meeting of the Vision Sciences Society, Sarasota, FL.

Peterson, M. S., Kramer, A. F., Wang, R. F., Irwin, D. E., \& McCarley, J. S. (2001). Visual search has memory. Psychological Science, 12, 287-292.

Shore, D. I., \& KLEIN, R. M. (2000). On the manifestations of memory in visual search. Spatial Vision, 14, 59-75.

Theeuwes, J., Kramer, A. F., Hahn, S., Irwin, D. E., \& Zelinsky, G. J. (1999). Influence of attentional capture on oculomotor control. Journal of Experimental Psychology: Human Perception \& Performance, 25, 1595-1608.

Toet, A., Bisl, P., Koor, F. L., \& Valeton, J. M. (1998). A high-resolution image dataset for testing search and detection models (TNO-NM-98A020). Soesterberg, The Netherlands: TNO Human Factors Research Institute.

Treisman, A. M., \& Gelade, G. (1980). A feature-integration theory of attention. Cognitive Psychology, 12, 97-136.

Wojciulik, E., Rorden, C., Clarke, K., Husain, M., \& Driver, J. (2004). Group study of an "undercover" test for visuospatial neglect: Invisible cancellation can reveal more neglect than standard cancellation. Journal of Neurology, Neurosurgery, \& Psychiatry, 75, 13561358.

Wolfe, J. M. (1994). Guided Search 2.0: A revised model of visual search. Psychonomic Bulletin \& Review, 1, 202-238.

Woodman, G. F., Vogel, E. K., \& LucK, S. J. (2001). Visual search remains efficient when visual working memory is full. Psychological Science, 12, 219-224.

\section{NOTES}

1. In the case of the scene-based experiments, the set of display positions eligible for fixation corresponded to the same 48 grid cells used to characterize the behavioral reinspection rates. To prevent the model from sampling improbable scene regions, the set of eligible grid cells was further constrained to those regions fixated by the observer in the corresponding behavioral data.

2 . Note that the model was prevented from immediately resampling objects (i.e., selecting the same object on fixation $i+1$ as on fixation $i$ ). As in the behavioral data, immediate refixations, cases in which an object was selected on fixation $i$, then reselected on fixation $i+2$, were also excluded from the simulated data. 
3. Further analysis of these Experiment 4 data revealed that misses were higher in the marking condition only for small targets $[24.2 \%$ vs. $10 \% ; t(9)=2.63, p<.05]$; miss rates for the large targets were substantially lower and not significantly different (1.7\% vs. $1.5 \%)$. We speculate that the markers were occasionally covering small targets and that this was contributing to the higher miss rate.

4. In a preliminary experiment investigating the possibility of marker onset costs, we also compared data from a marker onset condition with those from a marker offset condition in an O-Q search task (Dickinson,
Chen, \& Zelinsky, 2003). The procedure in the onset condition was similar to that described in the present Experiment 2, except for the markers being red boxes surrounding fixated objects. The offset condition presented a search display in which every object was initially surrounded by a red box. Objects were then marked by having this box disappear when gaze shifted away from a fixated object. Analyses of these data revealed no systematic differences between onset and offset conditions in either RTs or distractor refixations, further supporting our conclusion that marker onsets were not capturing attention and, thereby, introducing a cost.

\section{APPENDIX}

\section{Experiment 1}

The target was an $\mathrm{O}$ and the distractors were Q-like objects oriented at $0^{\circ}, 90^{\circ}, 180^{\circ}$, and $270^{\circ}$. The Os subtended $0.61^{\circ} \times 0.61^{\circ}$, and the "tail" of the distractors subtended $0.07^{\circ} \times 0.07^{\circ}$. The objects were arranged randomly, with a minimum center-to-center object spacing of $2.87^{\circ}$ and a minimum display center to object center spacing of $2.84^{\circ}$. Marking consisted of replacing gray unmarked objects with bright pink versions of the same objects, provided that gaze remained for at least $80 \mathrm{msec}$ within an object's $2.8^{\circ}$ bounding circle.

There were 600 trials per observer, evenly divided into two display conditions (marking and no-marking), two target conditions (present and absent), and set sizes ranging from 7 to 35 objects. Marking and no-marking trials were completed on separate days, and 32 practice trials preceded each condition.

\section{Experiment 2}

Fifty children's toys were selected from the HEMERA object database (Hemera Technologies); set size was fixed at 14 objects. The minimum center-to-center distance between objects was $3.38^{\circ}$, and the minimum distance from the display's center to the center of any object was $2.84^{\circ}$. Except for these constraints, object placement in the display was random. All the objects could fit inside a $2.36^{\circ} \times 2.36^{\circ}$ bounding box and were presented in color against a black background. Markers were red square frames that subtended $3.38^{\circ} \times 3.38^{\circ}$ and were $0.17^{\circ}$ thick. Marking required that gaze enter a $3.4^{\circ}$ bounding circle surrounding an object and remain in this region for at least $100 \mathrm{msec}$.

A target object was designated before each trial $(1 \mathrm{sec})$, followed by a search display that remained visible until the response. Target objects were not reused within a display condition; distractors were selected randomly from the remaining set of 49 objects. There was a 2:1 ratio of absent to present trials per condition, and there were 24 practice trials.

\section{Experiment 3}

Displays were color images taken from Where's Waldo picture books. Twenty-four target-absent scenes were created by selectively scanning and cropping picture book images. Target-present scenes were created by segmenting the Waldo target and inserting it into the target-absent images, using Adobe Photoshop Version 7.1. To randomize target placement, displays were divided into a 16-cell grid, and targets were assigned with equal frequency to each cell. Targets were scaled to look correct in each image, with target size averaging $3.6^{\circ} \times 1.7^{\circ}$. Markers were $1^{\circ}$ semitransparent purple squares. Locations were marked if a fixation exceeded $200 \mathrm{msec}$ in duration and was at least $1^{\circ}$ from the last marked location.

Each of the 24 scenes appeared four times (twice in each display condition), for a total of 96 trials, divided evenly between present and absent conditions. Note that scene and target presence were decorrelated so as to prevent anticipatory responses upon the second viewing of a given scene. Accuracy feedback was provided after every trial, and the observers were familiarized with the target before the experiment. There were 16 practice trials.

\section{Experiment 4}

Displays were 24 landscape scenes modified from the "Search 2" image database (Toet, Bijl, Kooi, \& Valeton, 1998). Target-present trials were created by inserting a large $\left(2^{\circ} \times 1.4^{\circ}\right)$ or a small $\left(1^{\circ} \times 0.7^{\circ}\right)$ tank target into these scenes, with both target types appearing equally often in the right or left half of the display. The marker was a red semitransparent square. All other procedural and design details were the same as those in Experiment 3.

(Manuscript received July 19, 2004; 